\title{
Programas de fortalecimiento de prácticas parentales: un aporte a la prevención de conductas externalizantes en preescolares
}

\author{
Training programs in parental skills: a contribution to the prevention \\ of the development of externalizing behaviors in preschool children
}

\author{
Pablo Vergara-Barra ${ }^{\mathrm{a}, \mathrm{c,d}}$, Paulina Rincón ${ }^{\mathrm{b}, \mathrm{d}}$, Karen Oliva-Jara ${ }^{\mathrm{b}, \mathrm{c}, \mathrm{d}}$, \\ Consuelo Novoa-Rivera ${ }^{\mathrm{b}, \mathrm{c}, \mathrm{d}}$, Camila Pérez-Huenteo ${ }^{\mathrm{b}, \mathrm{c}, \mathrm{d}}$
}

\author{
aDepartamento de Psiquiatría y Salud Mental, Facultad de Medicina, Universidad de Concepción. Chile \\ bDepartamento de Psicología, Facultad de Ciencias Sociales, Universidad de Concepción. Chile \\ cObservatorio de Parentalidad, Universidad de Concepción. Chile \\ dPsicólogo/a
}

Recibido: 24 de abril de 2019; Aceptado: 8 de septiembre de 2019

\section{¿Qué se sabe del tema que trata este estudio?}

El fortalecimiento de habilidades parentales es a nivel global una de las estrategias más efectivas para el tratamiento y prevención de las más frecuentes dificultades en salud mental infanto-juvenil, en particular, en el ámbito de trastornos del comportamiento en preescolares.

\section{¿Qué aporta este estudio a lo ya conocido?}

Refuerza la idea de avanzar en intervenciones parentales de tipo promocionales y preventivas como estrategias factibles de ser implementadas aportando a la necesidad de crear dispositivos en este tópico alineadas directamente con el Plan Nacional de Salud Mental en Chile.

\section{Resumen}

La presencia de conductas externalizantes en la infancia tiene importantes grados de continuidad temporal futura, y es un factor de riesgo de fracaso y abandono escolar, de conductas delictuales, abuso y dependencia de sustancias, de conductas suicidas y de otros trastornos psicopatológicos del desarrollo. En esta revisión se analizan los principales aspectos relacionados con los programas de fortalecimiento de prácticas parentales como dispositivos útiles en el marco de estrategias multicomponentes de trabajo preventivo. La evidencia ha demostrado que la intervención dirigida a madres y padres es crucial en la modificación de problemas de conducta en niños/as, siendo el entrenamiento en habilidades parentales una de las estrategias mejor estudiadas y consideradas de mayor calidad al momento de prevenir el desarrollo de conductas externalizantes.

Correspondencia:

Karen Oliva-Jara

karenoliva@udec.cl 


\section{Abstract}

The presence of externalizing behaviors in childhood has significant degrees of future self-continuity and is a risk factor for school failure and drop-out, criminal behavior, substance abuse and dependence, suicidal behaviors and other developmental psychopathological disorders. In this review, the main aspects related to parental practices strengthening programs are analyzed as useful instruments in the context of multi-component preventive work strategies. The evidence has shown that intervention aimed at mothers and fathers is crucial in the modification of behavioral problems in children, where training in parental skills is one of the most studied strategies and considered of higher quality when preventing the development of externalizing behaviors.

\section{Keywords:}

Externalizing

behaviors;

Parental training

programs;

Parenting

\section{Introducción}

Uno de los problemas relacionados con el desarrollo y salud mental de niños y niñas que más preocupación genera es la presencia de conductas disruptivas (agresividad, desobediencia acentuada y hostilidad) y la ausencia de respeto a normas de convivencia y a derechos de otras personas ${ }^{1}$. Estos comportamientos desadaptativos reciben la denominación genérica de problemas de conducta o conductas externalizantes². Si bien imprecisos, estos rótulos han recibido acogida pues permiten dar cuenta, sin implicar necesariamente la existencia de patología, de un conjunto de dificultades que son fuente de impedimento social y educacional que generan tensión y afectan no solo al bienestar de los niños y niñas que las presentan, sino que también a sus familias y a su entorno ${ }^{1}$. De acuerdo a los datos del consorcio Joint Effort Toddler Temperament (JEETC) un grupo importante de dificultades de salud mental en la infancia está relacionado con comportamiento desadaptativo externalizante ${ }^{3}$. En Chile los problemas de conducta según datos oficiales constituyen una de las más altas prevalencias y son uno de los principales motivos de consulta en los servicios de salud ${ }^{4,5}$.

Se ha demostrado que los programas de intervención dirigidos a madres y padres, tanto en prevención como en tratamiento, son cruciales en la modificación de conductas externalizantes en niños, siendo el entrenamiento en habilidades parentales una de las estrategias más estudiadas y con mejores resultados ${ }^{6,7}$. Los padres y/o cuidadores/as de niños que presentan conductas externalizantes muestran frecuentemente un estilo de crianza negativo y coercitivo ${ }^{8}$, que a partir de dichos programas puede ser modificado, beneficiando la evolución del diagnóstico. No es posible señalar que la hostilidad, como característica en la crianza, provoca la externalización (por ejemplo hiperactividad), ésta parece desempeñar un rol importante en su incremento y mantención ${ }^{9}$.

Si bien es un tema controversial, diversas guías clínicas recomiendan inicialmente intervenir con téc- nicas de manejo psicosocial y como último recurso el tratamiento farmacológico, considerando la opinión de expertos y no necesariamente en base a evidencia científica que avale superioridad de dichas intervenciones iniciales sobre el tratamiento medicamentoso ${ }^{10,11}$. Los especialistas sugieren proceder de este modo, para evitar los efectos colaterales de los fármacos que aún no se estudian en profundidad y pudiesen generar efectos sobre el desarrollo del cerebro de niños, en este período de la vida, y hacer uso de ellos sólo en casos específicos en que las intervenciones psicosociales no han dado resultados ${ }^{12}$.

La eficacia de programas de intervención psicosocial en preescolares ha sido comprobada en diversos contextos realizándose en estos las adaptaciones culturales apropiadas a la idiosincrasia de los lugares ${ }^{3,12}$. Se ha demostrado que realizar cambios a partir de la modificación del ambiente escolar y/o familiar del niño, genera un efecto positivo en la evolución negativa potencialmente disfuncional de la conducta externalizante ${ }^{13}$. Los programas de entrenamiento parental, especialmente en etapas tempranas de la conducta externalizante, resultan muy beneficiosos ${ }^{14}$. Pese a esto, recientes investigaciones sugieren que hay escasos estudios rigurosos en países de bajo y mediano ingreso económico ${ }^{15,16}$. Asimismo intervenciones tales como Psicoeducación, Terapia Interaccional Padre-Hijo/a (TIPH), Intervenciones conductuales en la sala de clases y, con menor frecuencia, el entrenamiento en autocontrol, también tienen resultados ventajosos ${ }^{17-19}$.

En base a los antecedentes expuestos el propósito de la presente revisión de literatura fue analizar los principales aspectos relacionados con los programas de fortalecimiento de prácticas parentales como dispositivos útiles en el marco de estrategias multicomponentes de trabajo preventivo.

Para dar respuesta a esto se hizo una búsqueda de artículos científicos y bibliografía especializada que da$\tan$ desde el año 2000 al año 2019 en las bases de datos PubMed, Web of Science y Scielo. Se llevó a cabo una revisión de tipo descriptiva de la literatura con el fin 
de extraer la información necesaria que se relacionara con el objetivo del presente trabajo. Los descriptores MESH en inglés utilizados para la búsqueda fueron: "parents programmes", "parental practices training" y "child externalizing behaviors"; en español se utilizaron los términos: "programas parentales", "prácticas parentales" y "conductas externalizantes". Los criterios de selección de los artículos escogidos fueron determinados por el objetivo de esta revisión de literatura y su calidad a través de una lectura crítica por parte del equipo de trabajo.

\section{Conductas externalizantes y disrupción del comportamiento}

Cuando las conductas externalizantes configuran patrones comportamentales disfuncionales acentuados y relativamente estables son considerados trastornos psicopatológicos. En la quinta edición del Manual Diagnóstico y Estadístico de Trastornos Mentales (DSM-5) las categorías diagnósticas relacionadas más directamente con las conductas externalizantes son las de Trastorno Negativista Desafiante, Trastorno de Conducta y Trastorno Disocial, insertos en el capítulo de Trastornos Destructivos del Control de Impulsos y de la Conducta ${ }^{20}$. Por otro lado, en la décimo primera versión de la Clasificación Internacional de Enfermedades (CIE-11) las conductas externalizantes engloban al Trastorno Oposicionista Desafiante y Disocial, en el capítulo de Trastornos Mentales, Conductuales o del Neurodesarrollo ${ }^{21}$.

Por un lado el DSM-5 considera que el Trastorno Oposicionista, el Trastorno Disocial y el Trastorno de Conducta son diagnósticos diferentes, por el otro la CIE-11, señala que todos ellos son manifestaciones en distinto grado de una misma disfuncionalidad. Si bien esto pudiera hablar de posturas muy distintas, también existe un punto de convergencia entre ellas. Ambas coinciden en la identificación de conductas bajo el concepto de oposicionismo desafiante y trastornos de conducta. Asimismo, las dos reconocen una serie de elementos en común para la denominación de conducta externalizante ${ }^{1}$. En este universo hay un grupo de niños con estos comportamientos que satisfacen los criterios categoriales de un trastorno de conducta; y hay otro que está en un umbral subclínico que no cumple con todos los criterios para ser considerado trastorno, sin embargo, representa un grupo de riesgo para el desarrollo del mismo ${ }^{22}$.

Respecto específicamente a los trastornos de conducta, a nivel internacional la mayoría de las investigaciones muestra que su prevalencia oscila en un rango de 5 a $10 \%$ en niños y niñas en edad escolar (datos que corresponden en su mayoría a estudios realizados en países desarrollados) $)^{1,23}$. En ese tiempo, usando criterios DSM-IV y basándose en el informe parental, la prevalencia de estos trastornos en nuestro país se estima en un $10 \%{ }^{5,24}$. Mientras menos disfuncionalidad y menos estresores psicosociales en edades tempranas menos son los costos que a nivel país implica trabajar con población adulta que cursa crónicamente con problemas de salud mental ${ }^{25}$. En estudios recientes se ha evidenciado que niños y niñas que han tenido problemas de conducta persistentes en el curso de la vida han utilizado significativamente más servicios en la adultez que aquellas personas adultas que en la infancia no han presentado dificultades de comportamiento, lo que señala altos costos futuros en términos de la utilización de la red de salud en múltiples sectores ${ }^{26}$. En un estudio de cohorte analizado por Rivenbark et al. (2018), el grupo con problemas de conducta en la infancia (con una prevalencia de 9\%), representó el 53,3\% de todas las condenas judiciales, el $15,7 \%$ de utilización de los servicios de urgencia, el 20,5\% de prescripción de medicamentos, el 13,1\% de las constancias por lesiones y el 24,7\% de los beneficios de asistencia social del país en etapas adultas ${ }^{25-27}$. El 50\% de las personas de este grupo en sus trayectorias de vida también acumuló un alto uso de los servicios del Estado en tres dominios: a) justicia penal; b) red de salud y c) servicios de bienestar social $^{26}$.

Sin negar la influencia de variables biológicas en el desarrollo de conductas externalizantes, existe consenso en la relevancia de factores psicosociales en la etiología de éstos y en su evolución ${ }^{28-30}$. La adversidad familiar ha mostrado sistemáticamente constituir un factor de riesgo de conductas externalizantes ${ }^{31}$. Del mismo modo, la presencia de discordia familiar, los conflictos de pareja y el déficit en los cuidados parentales tales como el comportamiento hostil hacia los hijos/as y la inconsistencia parental son las variables que se han mostrado relevantes para entender el origen y mantenimiento de los trastornos de conducta ${ }^{32-34}$.

La presencia de conductas externalizantes en la infancia tiene importantes grados de continuidad temporal futura, y es un factor de riesgo de fracaso y abandono escolar, de conductas delictuales, abuso y dependencia de sustancias, de conductas suicidas y de otros trastornos psicopatológicos del desarrollo ${ }^{30}$.

\section{Programas de entrenamiento en prácticas parentales}

La evidencia científica ha demostrado que la intervención dirigida a madres y padres, tanto en prevención como en tratamiento, es crucial en la modificación de problemas de conducta en niños, siendo el entrenamiento en habilidades parentales una de las 
estrategias mejor estudiadas y consideradas de mayor calidad $^{7}$. En este tipo de intervención -de carácter grupal- los elementos centrales de los programas de entrenamiento parental efectivos se focalizan en el desarrollo de capacidades para incrementar en los participantes interacciones positivas con los niños a su cuidado y la inclusión de habilidades para favorecer entre todos ellos la comunicación emocional ${ }^{14,35,36}$.

Los programas de entrenamiento parental, son una de las estrategias más investigadas en la intervención con conductas externalizantes, ya sea como componente exclusivo o en el marco de estrategias multicomponentes ${ }^{6,7,37,38}$. El meta-análisis de Furlong et al. (2012) de programas de entrenamiento parental mostró que estos disminuían las conductas problemáticas de niños y niñas así como las prácticas parentales "rudas" hacia ellos y ellas mejorando al mismo tiempo las competencias parentales, el bienestar y salud mental de las personas participantes ${ }^{7}$. En esta misma línea argumental en un reciente meta-análisis de Leijten et al. (2017) se informa que este tipo de programas reducen la inconsistencia parental, reducen el maltrato hacia niños y niñas (castigos corporales, amenazas, gritos) $\mathrm{y}$ aumentan la frecuencia de incentivos, halagos y re- fuerzos positivos desde los participantes hacia ellos y ellas $^{38}$.

A nivel internacional el proyecto Blueprints - especializado en la evaluación de programas promocionales y preventivos en el campo de la salud mental en la niñez y juventud- identifica 17 programas de entrenamiento parental (con distintos objetivos, formatos y población objetivo) como "modelos" o "promisorios" (las dos categorías de más alto nivel) ${ }^{39}$. Los programas de entrenamiento parental que aparecen reconocidos en el listado de Blueprints son Años Increíbles (Incredible Years $)^{40,41}$ y el Programa de Parentalidad Positiva Triple $\mathrm{P}^{42,43}$. Aún cuando no son los únicos programas visualizados, se destacan en ellos su diseminación a nivel internacional y referencia obligada cuando se alude a programas de entrenamiento parental en formato grupal ${ }^{39}$. En Chile existe un programa de carácter promocional y preventivo de tipo universal que fue probado en un ensayo clínico aleatorizado por conglomerados con muy buenos resultados: Día a Día $\mathrm{UdeC}^{6,44}$ (tabla 1). El éxito en términos de disminución de conductas externalizantes de preescolares y de mejoras en prácticas de crianza van en la misma línea de los programas reconocidos por Blueprints con óp-

Tabla 1. Aspectos centrales de los programas de entrenamiento parental

\begin{tabular}{|c|c|c|c|}
\hline Ítems & Años Increibles & $\begin{array}{l}\text { Programa de Parentalidad Positiva, } \\
\text { Triple P }\end{array}$ & Día a Día UdeC \\
\hline Descripción & $\begin{array}{l}\text { Serie de tres programas indepen- } \\
\text { dientes, de multifases diseñado para } \\
\text { promover competencias socioemo- } \\
\text { cionales y para prevenir, reducir y } \\
\text { tratar problemas de comportamiento } \\
\text { y emocionales en niños }\end{array}$ & $\begin{array}{l}\text { Programa multinivel (5) que busca } \\
\text { prevenir e intervenir en problemas } \\
\text { graves de comportamiento, emocio- } \\
\text { nales y del desarrollo de niños de } 0 \text { a } \\
16 \text { años a través de mejorar el cono- } \\
\text { cimiento y confianza de los padres }\end{array}$ & $\begin{array}{l}\text { Programa estructurado de entre- } \\
\text { namiento dirigido a las familias de } \\
\text { niños y niñas. El cual busca potenciar } \\
\text { en los y las participantes prácticas } \\
\text { parentales apropiadas para favorecer } \\
\text { el desarrollo de su hijo o hija en edad } \\
\text { preescolar y evitar el desarrollo de } \\
\text { comportamientos y trastornos exter- } \\
\text { nalizados }\end{array}$ \\
\hline Modalidad & Grupal & $\begin{array}{l}\text { Nivel Grupal } \\
\text { Nivel individual } \\
\text { Nivel auto dirigido }\end{array}$ & Grupal \\
\hline Público objetivo & $\begin{array}{l}\text { Padres, madres y/o cuidadores de } \\
\text { personas entre } 3 \text { a } 8 \text { años }\end{array}$ & $\begin{array}{l}\text { Padres, madres y/o cuidadores de } \\
\text { personas entre } 0 \text { a } 16 \text { años }\end{array}$ & $\begin{array}{l}\text { Padres, madres y/o cuidadores de } \\
\text { personas entre } 3 \text { a } 5 \text { años }\end{array}$ \\
\hline Número de sesiones & $\begin{array}{l}\text { Programa básico } 12 \text { sesiones } \\
\text { Programa avanzado } 20 \text { a } 24 \text { sesiones }\end{array}$ & $\begin{array}{l}\text { Sesiones grupales pueden variar entre } \\
8 \text { a } 10 \text { sesiones }\end{array}$ & 6 sesiones \\
\hline Componentes & $\begin{array}{l}\text { Juego dirigido por el niño } \\
\text { Apoyo académico } \\
\text { Entrenamiento social y emocional } \\
\text { Elogios y programas de incentivos } \\
\text { Rutinas y reglas } \\
\text { Técnicas de disciplina positiva }\end{array}$ & $\begin{array}{l}\text { Componentes de la crianza positiva } \\
\text { Entorno seguro } \\
\text { Promover un ambiente de aprendi- } \\
\text { zaje positivo } \\
\text { Disciplina positiva } \\
\text { Cuidado del padre } \\
\text { *Los componentes varían según el } \\
\text { nivel }\end{array}$ & $\begin{array}{l}\text { Comunicación afectiva } \\
\text { Juego dirigido por niños y niñas } \\
\text { Ordenes, rutinas y transiciones } \\
\text { Atender e ignorar planificadamente } \\
\text { Refuerzos y programa de incentivos } \\
\text { Consecuencias lógicas y naturales } \\
\text { Tiempo Fuera de Reforzamiento } \\
\text { Positivo }\end{array}$ \\
\hline País & Estados Unidos de América & Australia & Chile \\
\hline
\end{tabular}

Fuente elaboración propia basado en las referencias 6, 40-44 
timos niveles de efectividad, altos estándares de calidad y reducción potencial de maltrato infantil ${ }^{6,40-44}$.

\section{Conclusiones}

Los problemas de conducta en etapas iniciales del desarrollo siguen constituyendo una de las problemáticas de salud mental de más alta prevalencia en niños y niñas y de los principales motivos de consulta en los servicios de salud mental infanto-juvenil. Actualmente, con las reformulaciones en los criterios diagnósticos, la conducta externalizante y su grado de contuinidad futura representa un constructo amplio que incorporaría a grupos de niños y niñas que se contabilizarían tanto por criterios clínicos como subclínicos. Si bien la etiología de las conductas externalizantes se puede asociar a múltiples factores, la literatura sugiere que las prácticas parentales son uno de los elementos más relevantes en el desarrollo de ellas en la infancia. En este contexto los programas de fortalecimiento en habilidades parentales aparecen como dispositivos válidos de prevención e intervención siempre que consideren un diseño contextualizado a la realidad sociocultural en la que se lleven a cabo. La metodología de trabajo en los programas efectivos es de carácter grupal con un alto componente de promoción de habilidades prácticas y vivenciales. Contempla estrategias andragógicas con el fin de mejorar la participación y adherencia a los protocolos de los dispositivos situando a los participantes como expertos por experiencia.

\section{Conflicto de intereses}

Los autores declaran no tener conflicto de intereses.

\section{Agradecimientos}

Proyecto Fondo de Fomento al Desarrollo Científico y Tecnológico FONDEF ID 14I20058, Chile. Instituto Milenio para la Investigación en Depresión y Personalidad MIDAP, Chile. Programa de Doctorado en Salud Mental y Programa de Doctorado en Psicología de la Universidad de Concepción.

\section{Financiamiento}

Becas Doctorado Nacional CONICYT Folios: 21180292, 21180983, 21181101, 21170272.

\section{Referencias}

1. Moffitt TE, Scott S. Conduct disorders of childhood and adolescence. Rutter's Child and Adolescent Psychiatry, $5^{\text {th }}$ ed: WileyBlackwell; 2008. p. 543-64.

2. Achenbach TM, McConaughy SH, Ivanova MY, Rescorla LA. Manual for the ASEBABrief Problem Monitor (BPM). Burlington: VT ASEBA; 2011.

3. Gartstein M, Putnam S. Toddlers, parents and culture: Findings from the Joint Effort Toddler Temperament Consortium. New York: Routledge; 2019.

4. Vicente B, Saldivia S, De la Barra F, Melipillán R, Valdivia M, Kohn R. Prevalence of psychiatric disorders among Chilean children and adolescents. Rev Med Chile 2012;140(4):447-57.

5. Vicente B, Saldivia S, Pihán R. Prevalencias y brechas hoy: salud mental mañana. Acta Bioethica 2016;22(1):51-61.

6. Rincón P, Cova F, Saldivia S, et al. Effectiveness of a positive parental practices training program for Chilean preschoolers' families: a randomized controlled trial. Front Psychol. 2018; 9:175.

7. Furlong M, Mcgilloway S, Bywater T, et al. Behavioural and cognitive-behavioural group-based parenting programmes for early-onset conduct problems in children aged 3 to 12 years (Review). Cochrane Database of Systematic Reviews. [Internet]. 2012;(2):1-357. Available from: https://www.cochranelibrary.com/ cdsr/doi/10.1002/14651858.CD008225. pub2/epdf/full [citado el 13 de septiembre de 2018].

8. González R, Bakker L, Rubiales J. Estilos parentales en niños y niñas con TDAH. Rev.latinoam.cienc.soc.niñez juv 2014;12(1):141-58.

9. Sinclair M. Behavioural and cognitive approaches. People with hiperactivity: understanding and managing their problems. Mac Keith Press; 2008. p. 14259.

10. National Collaborating Centre for Mental Health (UK). NICE Guideline N ${ }^{\circ} 87$ Attention Deficit Hyperactivity Disorder Diagnosis and Management of ADHD in Children, Young People and Adults. Leicester (UK); 2018.

11. Ministerio de Sanidad Política Social e Igualdad. Guía de Práctica Clínica sobre el Trastorno por Déficit de Atención con Hiperactividad (TDAH) en niños y adolescentes. Barcelona; 2010.

12. Serrano-Troncoso E, Guidi M, Alda-Diez JA. Is psychological treatment efficacious for attention deficit hyperactivity disorder (ADHD)? Review of non-pharmacological treatments in children and adolescents with ADHD. Actas Esp de Psiquiatr 2013;41(1):44-51.

13. Palacio JD, Ruiz-García M, Bauermeister JJ, Montiel-Navas C, Henao GC, Agosta G. Algoritmo de tratamiento multimodal para preescolares latinoamericanos con Trastorno por Déficit de Atención con Hiperactividad (TDAH). Salud Mental 2009;32(1):3-16.

14. Lozano-Rodríguez, I; Valero-Aguayo L. Una revisión sistemática de la eficacia de los programas de entrenamiento a padres. Revista de Psicología Clínica con Niños y Adolescentes 2017;4(2):85-91.

15. Knerr W, Gardner F, Cluver L. Improving positive parenting skills and reducing harsh and abusive parenting in low- and middle-income countries: a systematic review. Prevention Science 2013;14(4):352-63.

16. Mejia A, Calam R, Sanders MR. A review of parenting programs in developing countries: opportunities and challenges for preventing emotional and behavioral difficulties in children. Clin. Child Fam. Psychol. Rev 2012;15(2):163-75.

17. Ferrín M, Taylor E. Child and caregiver issues in the treatment of attention 
deficit-hyperactivity disorder: education, adherence and treatment choice. Future Neurology 2011;6:399-413.

18. Van den Hoofdakker BJ, Van der VeenMulders L, Sytema S, et al. Effectiveness of behavioral parent training for children with ADHD in routine clinical practice: a randomized controlled study. J Am Acad Child Adolesc Psychiatry. 2007;46(10):1263-71.

19. Webster-Stratton C, Jamila Reid M, Stoolmiller M. Preventing conduct problems and improving school readiness: evaluation of the Incredible Years Teacher and Child Training Programs in highrisk schools. J. Child Psychol. Psychiatry 2008;49:471-88.

20. Asociación Americana de Psiquiatría (APA). Manual diagnóstico y estadístico de los trastornos mentales DSM V ( $5^{\mathrm{a}}$ ed.). Arlington VA, Estados Unidos: American Psychiatric Publishing; 2013.

21. Organización Mundial de la Salud (OMS). Clasificación Internacional de Enfermedades CIE-11 [Internet]. 2018; [citado el 22 de noviembre de 2018]. Disponible en: https:/icd.who.int/en/

22. Frick PJ, Morris AS. Temperament and developmental pathways to conduct problems temperament and developmental pathways to conduct problems. J. Clin. Child Adolesc. Psychol 2004;33:37-41.

23. Rescorla L, Achenbach T, Ivanova MY, et al. International comparisons of behavioral and emotional problems in preschool children: parents' reports from 24 societies. J. Clin. Child Adolesc. Psychol 2011;40(3):456-67.

24. Vicente B, Saldivia S, De La Barra F, et al. Prevalence of child and adolescent mental disorders in Chile: a community epidemiological study. J Child Psychol Psychiatry. 2012;53(10):1026-36.

25. Schaefer JD, Caspi A, Belsky DW, et al. Enduring mental health prevalence and prediction. J Abnorm Psychol. 2017;126(2):212-24.

26. Rivenbark JG, Odgers CL, Caspi A, et al. The high societal costs of childhood conduct problems: evidence from administrative records up to age 38 in a longitudinal birth cohort. J. Child Psychol. Psychiatry 2018;59(6):703-10.

27. Caspi A, Houts RM, Belsky DW, et al. Childhood forecasting of a small segment of the population with large economic burden. Hum. Nat 2016;1:5.

28. Tremblay RE. The development of agressive behaviour during childhood: what have we learned in the past century? Int. J. Behav. Dev 2000;24(2):129-41.

29. Pingault JB, Côté SM, Galéra C, et al. Childhood trajectories of inattention, hyperactivity and oppositional behaviors and prediction of substance abuse/ dependence: a 15-year longitudinal population-based study. Mol Psychiatry. 2013;18(7):806-12. Available from: https:// www.nature.com/articles/mp201287.

30. Petersen IT, Bates JE, Dodge KA, et al. Describing and predicting developmental profiles of externalizing problems from childhood to adulthood. Dev. Psychopathol 2015;27(3):791-818.

31. Cova F, Maganto C, Melipillán R. Género, adversidad familiar y síntomas emocionales en preadolescentes. Psykhe (Santiago) 2005;14(1):227-32.

32. Cova F. La psicopatología evolutiva y los factores de riesgo y protección: el desarrollo de una mirada procesual. Revista de Psicología 2004;13(1):93-102.

33. Price J, Chiapa A, Escobar N. Predictors of externalizing behavior problems in early elementary-aged children: the role of family and home environments. J Genet Psychol. 2013;174(4):464-71.

34. Ramírez A. Conflictos entre padres y desarrollo de los hijos. Revista de Ciencias Sociales 2003;11(34):171-82.

35. Robles Z, Romero E. Programas de entrenamiento para padres de niños con problemas de conducta: una revisión de su eficacia. Anales de Psicología 2011;27(1):86-101.

36. Kaminsky J, Valle LA, Filene JH, et al. A meta-analytic review of components associated with parent training program effectiveness. J Abnorm Child Psychol. 2008; 36(4):567-89.

37. Lundahl B, Risser HJ, Lovejoy MC. A meta-analysis of parent training: moderators and follow-up effects. Clin. Psychol. Rev 2006;26(1):86-104.

38. Leijten P, Gardner F, Landau S, et al. Research review: harnessing the power of individual participant data in a metaanalysis of the benefits and harms of the Incredible Years parenting program. J Child Psychol Psychiatry. 2017;59(2):99109.

39. Center for the Study and Prevention of Violence Institute of Behavioral Science University of Colorado Boulder. Blueprints for healty and youth development. [Internet] [citado el 13 de diciembre de 2018]. Available from: https://www. blueprintsprograms.org/programs

40. Webster-Stratton C, Reid J, Hammond M. Social skills and problem solving training for children with early onset conduct problems: Who benefits? J Child Psychol Psychiatry 2001;42(7):943-52.

41. Webster-Stratton, C. (2000). The incredible years training series (pp. 1-24). Washington, DC: US Department of Justice, Office of Justice Programs, Office of Juvenile Justice and Delinquency Prevention.

42. Sanders M, Baker S, Turner K. A randomized controlled trial evaluating the efficacy of Triple P Online with parents of children with early-onset conduct problems. Behav Res Ther. 2012; 50(11):675-84.

43. De Graaf I, Speetjens P, Smit F, et al. Effectiveness of the Triple P Positive Parenting Program on behavioral problems in children: A meta-analysis. Behav Modif. 2018; 32(5): 714-735.

44. Cova F, Rincón P, Bustos C, et al. Randomized cluster trial of a parenting program in Chile: Key mediators in the decrease in behavior problems in preschool children. Clin Child Psychol P. 2019;1(1):1-13. 